\title{
Rôles et stratégies paternels dans une entreprise familiale artisanale : exemple des imprimeurs à Venise (deuxième moitié XVe-début XVIe siècles)
}

\author{
Catherine Kikuchi
}

Johann de Spire, «qui a côtoyé de nombreuses autres villes avant de s'établir [à Venise], où il habite avec femme, enfants et toute sa famille, et où il exerce cet art d'imprimer des livres ${ }^{1} \gg$, est le premier imprimeur établi à Venise. En 1469, il obtient de la Seigneurie un privilège de cinq ans sur la nouvelle technique. Le texte de ce privilège nous le présente explicitement comme un père de famille, à la tête d'une entreprise typographique, à laquelle les différents membres de sa maisonnée mentionnés ici participent certainement. Il obtient ce privilège en tant qu'imprimeur allemand, arrivé à Venise pour apporter l'art d'imprimer inconnu jusqu'alors, mais également en tant que chef d'une entreprise familiale à part entière, qui garantit sa stabilité dans la ville et sa réussite. Johann de Spire meurt un an plus tard, mais à sa suite viennent s'installer de nombreux autres imprimeurs dans la lagune, si bien que Venise devient rapidement le premier pôle de l'imprimerie naissante en Europe.

Les grandes dynasties familiales sont celles qui ont fait la célébrité et la prospérité de l'imprimerie en Europe. À Paris, on pense à la famille des Estienne; à Louvain, à celle des Plantin; à Venise, la dynastie des Manuce transmet sur trois générations un savoir-faire et une réputation d'imprimeurs humanistes. L'entreprise familiale apparaît à première vue comme une forme d'organisation du travail courante pour les imprimeurs. En réalité, celle-ci est une configuration sociale loin d'être acquise.

L'entreprise familiale, Familienbetrieb, ou encore family business, a déjà fait l'objet d'études approfondies depuis les années $1980^{2}$. Cette organisation du travail est notamment définie par Michael Miterrauer en 1984 à partir de deux aspects : une entreprise qui perdure par transmission entre les générations à travers l'héritage; et l'interaction économique de personnes vivant dans le même ménage. Nous nous intéresserons ici conjointement à ces deux perspectives, qui concernent directement le rôle du père en ce qu'il transmet l'héritage et dans le même temps dirige le travail familial.

Des travaux ont évoqué de façon plus ou moins approfondie le rôle de la transmission du capital matériel dans le contexte de l'imprimerie naissante à Venise. La succession des générations a bien été soulignée dans les travaux concernant les toutes premières entreprises d'imprimerie, menés notamment par Philippe Braunstein ${ }^{3}$. Cependant, la famille, et encore moins la paternité, n'ont jamais été étudiées en tant que tels dans le cadre de l'imprimerie, alors qu'elles jouent un rôle primordial dans ce contexte économiquement difficile et socialement spécifique.

En effet, jusqu'au milieu du XVI ${ }^{\mathrm{e}}$ siècle à Venise, les imprimeurs se situent en dehors de tout métier organisé : les régulations sont donc moins nombreuses et moins contraignantes, notamment dans ce qui concerne l'organisation du travail ou l'apprentissage. Cette activité nécessite de plus un capital matériel et financier important : les presses, mais surtout les caractères typographiques représentent un investissement conséquent, et la rentabilité des premières éditions réalisées n'était jamais garantie. Enfin, le milieu du livre à Venise est marqué par une très forte proportion d'étrangers, en particulier des Allemands, mais également des Français, des Anglais, et des Italiens hors du Domaine vénitien.

\footnotetext{
$1 \quad$ Archivio di Stato di Venezia (ASV), Collegio, Notatorio, reg. XIX, 1467-73, c 55v, je traduis.

2 G. ALFANI (dir.), Il Ruolo economico della famiglia, Rome, Bulzoni, 2006.

3 P. BRAUNSTEIN, «Les Allemands et la naissance de l'imprimerie vénitienne », Revue d'études italiennes, 1981, vol. 27, n 4, p. 381-389.
} 
Il s'agit ici de comprendre comment la paternité peut servir comme révélateur de l'intégration sociale et économique du nouveau groupe artisanal que constituent les imprimeurs à Venise. L'étude de ce cas particulier permettra également de mieux comprendre les évolutions du rôle du père dans les sociétés urbaines de la fin du Moyen Âge.

Je prendrai ici «père » dans un sens plus large que seulement biologique. De nombreux historiens ont bien souligné que la famille au Moyen Âge comprenait aussi bien le noyau familial composé des parents et des enfants, mais également des apprentis, domestiques, et parents plus ou moins proches qui font partie de la maisonnée. Michael Mitterauer, cité plus haut, affirmait dans le même article que l'on ne pouvait pas, au Moyen Âge, introduire une distinction entre famille et ménage ou maisonnée, entendue comme ensemble des personnes vivant sous un même toit ${ }^{4}$. Ici, $\mathrm{j}$ 'entendrai donc le père de famille comme chef de cette maisonnée ${ }^{5}$.

\section{Un contexte spécifique pour la filiation : évolutions chronologiques}

Le statut de père de famille est donc affirmé dans le premier document officiel que nous avons concernant l'imprimerie à Venise, le privilège que le conseil accorde à Johann de Spire en 1469. Cependant, cette situation familiale stable est une exception parmi les imprimeurs qui l'ont suivi.

Les imprimeurs à Venise des années 1470 sont pour l'essentiel des étrangers non-italiens, surtout des Allemands : entre 1469 et 1479, moins de $40 \%$ des imprimeurs et éditeurs exerçant à Venise sont d'origine italienne; près de $50 \%$ sont d'origine germanique dans les cinq premières années ${ }^{6}$. Il est difficile de déterminer s'ils étaient déjà présents depuis longtemps à Venise ; dans la plupart des cas, il est probable qu'ils soient arrivés dans la ville sans y avoir d'attaches particulières. Ainsi, Nicolas Jenson, français, est installé dans la lagune seulement depuis 1469, après avoir été formé à l'imprimerie à Mayence. Il s'agit d'une immigration de première génération, relativement récente et circonstancielle, d'artisans très qualifiés.

Cette immigration va de pair avec une relative précarité du fait de leur statut d'extranéité, mais également du fait de l'instabilité inhérente à l'activité. Sur l'ensemble de la période incunable, entre 1469 et 1500, $52 \%$ des typographes et éditeurs ayant imprimé un ouvrage à Venise arrêtent leur activité au bout de moins d'un an?

\section{Les premiers temps de l'imprimerie : la transmission sans fils}

C'est dans ce contexte qu'il faut étudier le statut paternel et la mention ou non d'enfants de ces premiers imprimeurs. En effet, dans les testaments de cette période, il n'est que très rarement fait mention d'enfants légitimes. On peut en particulier étudier le cas de Nicolas Jenson et de ses associés, dont les testaments nous sont parvenus et s'étalent entre 1474 et 1494. Ainsi, le testament de Nicolas Jenson en 1480 fait mention de plusieurs enfants naturels : Joanna, Catarina, Barbara, «filles naturelles du testateur», et Nicolas, «fils naturel du testateur». Aucune épouse légitime n'est mentionnée ${ }^{8}$. Un marchand qui lui est associé, Johann Rauchfass en 1478, fait également état d'enfants naturels, mais pas d'enfants légitimes, ni d'épouse reconnue'. Le testament de 1474 de Johann

4 M. MITERRAUER, «Familie und Arbeitsorganisation in städtischen Gesellschaften des Mittelalters und der frühen Neuzeit», A. HAVERKAMP (dir.), Haus und Familie in der Spätmittelalterlichen Stadt, Cologne, Böhlau, 1984, p. 9.

$5 \quad$ Nous exclurons de notre propos la parenté spirituelle; les sources qui nous renseignent sur les relations de parrainage sont encore trop rares pour en tirer des conclusions générales.

6 C. KIKUCHI, «Des Vagabonds des lettres? Les typographes à Venise aux temps des débuts de l'imprimerie », Précarité, instabilité, fragilité au Moyen Âge, journée d'étude du groupe Questes 14-15 juin 2013, Paris, à paraitre aux PUPS.

$7 \quad$ Ibid.

8 «filias naturales ipsius testatoris », «filio naturale ipsius testatoris »: ASV, Notarile, Testamenti, b. $68, n^{\circ} 263$, je traduis.

$9 \quad$ ASV, Notarile, Testamenti, b. 66, $\mathrm{n}^{\circ} 407$. 
Manthem, associé de Nicolas Jenson depuis 1480, ne mentionne aucun enfant ${ }^{10}$; de même pour le testament de deux autres de leurs partenaires, Johann Herborth en $1484^{11}$ et Hermann Liechtenstein en $1494^{12}$. Le cas de Jean de Spire, père d'une famille légitime, semble donc exceptionnel pour la période.

Cette situation est à lier directement au statut instable d'immigrant. On suppose que ces imprimeurs nouvellement immigrés à Venise étaient relativement jeunes, célibataires pour la plupart, et n'avaient pas les moyens de fonder un foyer, et ce d'autant plus qu'ils pouvaient faire faillite rapidement. Pour des raisons financières ou d'opportunisme, ils pouvaient quitter Venise quelques mois ou quelques années seulement après leur arrivée, installer leurs presses dans une autre ville, ou tout simplement changer d'activité. En effet, certains n'avaient pas l'intention de s'installer de façon définitive à Venise et menaient une carrière d'imprimeur itinérant ou semi-itinérant. De ce fait, les migrations sont nombreuses et fréquentes, qu'elles soient voulues ou contraintes. Ainsi Hermann Liechtenstein a été imprimeur à Vicence en 1475, à Trévise en 1477 puis enfin à Venise, suivant les opportunités qui se sont offertes à lui ${ }^{13}$. L'imprimeur français Jacques Le Rouge quitte Venise en 1478 pour la région de Turin, à la suite de la crise de surproduction qui touche l'imprimerie vénitienne ; auparavant, il avait été imprimeur à Rome sans doute à partir de 1470, puis à Pinerole en 1475-1476; après avoir quitté définitivement Venise, on le retrouve à nouveau à Pinerole entre 1478 et 1482 et enfin à Embrun en Savoie en $1489^{14}$.

L'instabilité et la mobilité sont des facteurs importants à prendre en compte qui expliquent largement que ces imprimeurs ne soient pas mariés et n'aient pas d'enfants légitimes. Le cas des enfants illégitimes était sans doute plus courant, à l'instar de Johann Rauchfass et de Nicolas Jenson. Johann Rauchfass est un marchand originaire de Francfort installé à Venise, qui investit dans le marché du livre par l'intermédiaire de la compagnie de Jenson. Il cite dans son testament de 1478 trois enfants naturels qu'il a eu avec une certaine Margarita, à qui il lègue de l'argent et qui semble avoir partagé sa vie à Venise ${ }^{15}$. D'autre part, Nicolas Jenson semble bien avoir associé son fils illégitime à l'activité de la compagnie ; il l'emploie à des activités commerciales à l'étranger mais son testament ne témoigne pas d'une volonté de lui léguer la suite de l'entreprise : le legs qui lui est destiné est élevé, mais il s'agit d'argent, et non de capital matérie ${ }^{16}$. Le statut d'enfant illégitime semble prévenir la volonté paternelle de transmettre en ligne directe l'entreprise typographique.

En l'absence d'enfants, les personnes légataires faisant partie de la famille sont essentiellement des frères, des sœurs, des neveux ou des nièces. Ainsi en 1474, Johann Manthem divise son patrimoine en cinq parts entre ses frères et sœurs et une nièce ${ }^{17}$. Dans le cas de Johann Herborth, des neveux sont les héritiers universels ${ }^{18}$. Enfin, dans le cas de Hermann Liechtenstein, c'est son neveu Peter Liechtenstein qui est héritier universel, recevant en particulier le matériel typographique de son oncle ${ }^{19}$.

Ces transmissions de patrimoine, qui sont en un sens perturbées par l'absence de fils, donnent lieu à des circulations de capital inhabituelles. Le capital matériel de l'imprimerie va avant tout aux personnes les plus proches qui ont appris le métier d'imprimeur. Cela peut être une personne de la

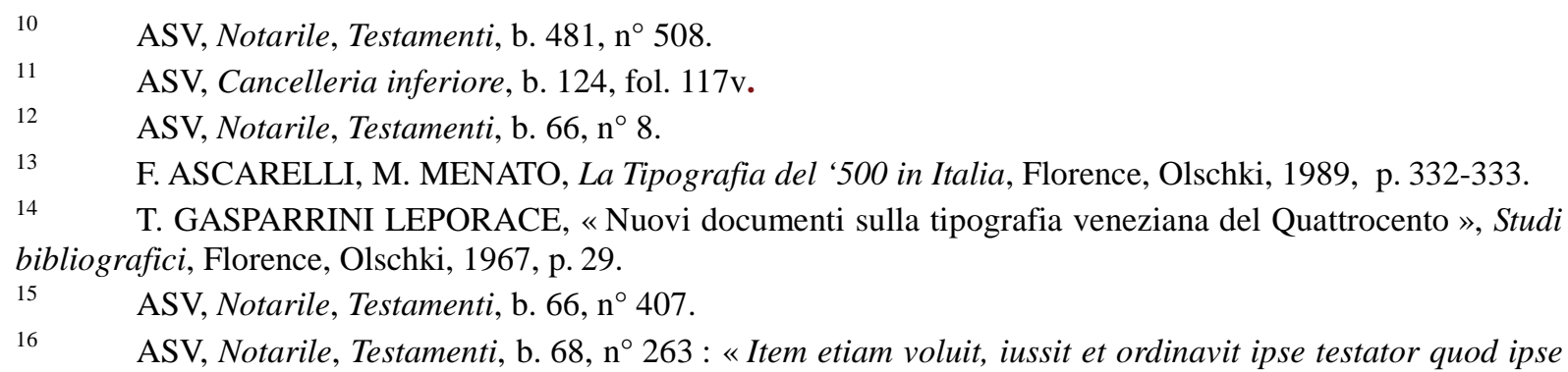
Albertus frater suus dare et consignare debeat Nicolao, filio naturali testatoris, in presenti habitatori lugduni, ducatos quadringentos auri, quando ipse filius suus pervenerit ad etatem annorum vigintiquinque, si et in quantum ipse filius suus se bene regeret et gubernaret $»$.

$17 \quad$ ASV, Notarile, Testamenti, b. 481, $\mathrm{n}^{\circ} 508$.

18 ASV, Cancellaria inferiore, Notai, b. 124, fol 117v.

19 ASV, Notarile, Testamenti, b. 66, n 8. 
famille, comme pour les Liechtenstein, mais également des apprentis ou des associés. Ainsi, les types de Jenson sont légués par testament à son ami et collaborateur, Peter Ugelheimer. Cependant, celui-ci n'est pas imprimeur, bien qu'il ait largement investi dans le marché du livre. C'est sans doute pour cette raison que les types de Jenson sont ensuite vendus à l'imprimeur Andrea Torresani ${ }^{20}$, un partenaire qui avait peut-être fait son apprentissage dans l'atelier de Jenson ${ }^{21}$. L'objectif est bien ici de faire en sorte que tout l'investissement productif effectué par les partenaires revienne entre des mains capables de le faire fructifier.

\section{L'émergence progressive d'une entreprise familiale}

Pendant la première époque de l'imprimerie à Venise, il n'est donc pas encore possible de parler d'entreprise familiale dans le sens d'une entreprise qui perdure de génération en génération. L'entreprise devient proprement familiale à partir du moment où la mention d'enfants légitimes et la transmission de l'héritage en ligne directe se généralisent pour les entreprises les plus prospères et dans les testaments que nous avons pu retrouver. Par exemple, Melchior Sessa hérite en 1505 de l'atelier de son père, Giovanni Battista Sessa et poursuit l'activité familiale ${ }^{22}$. De même Alessandro Paganino, fils illégitime de Paganino de Paganini, est l'héritier universel de son père, depuis le testament de 1505 de Paganino de Paganini, jusqu'à son dernier connu en $1538^{23}$. Il y met toutefois une condition : si son fils est incapable ou inapte à obtenir un tel héritage, ce sera son fils, Gaspar, petit-fils de Paganino, qui en héritera, ainsi que ses autres fils s'il en a. Alors que Nicolas Jenson avait choisi de ne pas transmettre son entreprise ou le matériel à son fils illégitime, Paganino de Paganini au contraire s'assure que son fils puisse reprendre à son compte l'atelier, à supposer qu'il en soit digne et capable : à ce moment-là, la continuité de l'entreprise est devenue plus importante que l'origine légitime ou non du fils. Cette situation semble avoir été acceptée par Cristina, l'épouse de Paganino et la belle-mère d'Alessandro, puisque celle-ci lui lègue cinquante ducats dans son premier testament de $1490^{24}$; Paganino en fait également la tutrice de son fils qui doit lui obéir en cas de décès de son père, comme s'il s'agissait de sa propre mère ${ }^{25}$. Cette situation témoigne bien du fait que le père met en œuvre des stratégies explicites, à travers ses relations familiales et ses dispositions testamentaires notamment, pour assurer la transmission de son patrimoine et de son savoir-faire à son fils.

L'évolution par rapport à la situation des débuts de l'imprimerie à Venise s'explique par une présence étrangère de deuxième génération ou de moins longue distance, puisque les Italiens sont désormais prédominants parmi les imprimeurs vénitiens. Pour reprendre les exemples précédemment cités, Giovanni Battista Sessa vient de la ville de Sessa, près de Lugano ${ }^{26}$; quant à Paganino Paganini, il vient de Brescia $^{27}$ et sa femme Cristina Fontana est la fille de Franciscus Renner, imprimeur d'origine allemande ayant déjà exercé à Venise entre 1473 et $1478^{28}$.

Le fait de voir apparaître les enfants dans les testaments est également le signe d'une intégration plus grande de l'imprimeur dans la ville et d'une relative stabilité géographique et économique. À partir des années 1490, il ne s'agit plus seulement d'entreprises qui lient des associés, plus ou moins

20 F. ASCARELLI, M. MENATO, La Tipografia del '500 in Italia, op. cit., p. 333.

21 A. NUOVO, Il Commercio librario nell'Italia del Rinascimento, Milan, FrancoAngeli, 1998, p. 76.

22 S. CURI NICOLARDI, Una Società tipografico-editoriale a Venezia nel secolo XVI: Melchiorre Sessa e Pietro di Ravani (1516-1525), Florence, Olschki, 1984, p. 6.

23 A. NUOVO, Alessandro Paganino (1509-1538), Padoue, Editrice Antenore, 1990, p. 242-247 : ASV, Notarile, Testamenti, b. 1216, v. IV, fol. 69v-70v ; ASBs, Notai di Salo, G. Golosino, fol. 41, Liber secundus, fol. 62 .

$24 \quad$ ASV, Notarile, Testamenti, b. 1153, n 22.

25 ASV, Notarile, Testamenti, b. 1216, vol. IV, fol. 69v-70.

26 F. ASCARELLI, M. MENATO, La Tipografia del '500 in Italia, op. cit., p. 327.

27 A. NUOVO, Alessandro Paganino, op. cit., p. 238 ; sans doute de Cigole, dans la campagne de Brescia.

28 R. RIDOLFI, «Francesco della Fontana, stampatore e libraio a Venezia », dans Studi bibliografici, Florence, Olschki, 1967. 
récemment arrivés dans la ville, mais d'entreprises proprement familiales, où les enfants sont espérés afin de faire perdurer l'entreprise.

Selon Michael Mitterauer, la transmission du patrimoine aux enfants est plutôt l'exception que la norme pour les entreprises familiales au Moyen Âge. Celle-ci dépend de trois conditions: la transmission au fils est plus fréquente quand les moyens de production coûtent chers, quand ils se trouvent dans un lieu précis et dépend aussi des conditions d'immigration ${ }^{29}$. Les trois critères se trouvent réunis ici à partir de la fin du $\mathrm{XV}^{\mathrm{e}}$ siècle. C'est donc sans surprise que les fils deviennent les héritiers universels, à partir du moment où on les retrouve systématiquement dans les testaments.

Pourtant, il serait faux de dire que la transmission de l'entreprise typographique aux enfants est une généralité à la fin $\mathrm{du} \mathrm{XV}^{\mathrm{e}}$ siècle à Venise. La majorité des ateliers dont nous avons la trace par les colophons ne se poursuit pas sur plusieurs générations et nous ne disposons pas d'assez de sources pour comprendre les mécanismes des héritages pour toutes ces entreprises. Par ailleurs, les neveux et les cousins continuent malgré tout à jouer un rôle important dans bon nombre de familles et d'entreprises. Il s'agit d'héritiers de secours : ils font souvent partie des légataires privilégiés en l'absence de fils. Les oncles jouant un rôle particulièrement important dans l'éducation de leurs neveux, on peut supposer que la relation qui les unit se rapproche de la relation père-fils. Ils remplaçaient les fils inexistants à la fois dans la transmission du capital, mais aussi dans la transmission du savoir-faire du père. On a ainsi l'exemple de la famille Giolito, dont le premier représentant imprimeur à Venise est Bernardino Stagnino. On retrouve dans la première moitié du XVI siècle des imprimeurs de la même famille : Giovanni Giolito, sans doute le neveu de Bernardino, puis Gabriele Giolito, qui obtiennent sans doute l'un après l'autre les instruments typographiques de Stagnino $^{30}$.

L'adaptation des stratégies du père de famille selon les circonstances familiales sont bien visibles dans le cas des deux testaments d'Alde Manuce, en 1506 et en $1514^{31}$. Dans le cas du premier, Alde est déjà marié et son épouse est enceinte pour la première fois. Les dispositions testamentaires signalent que la venue d'un fils était attendue et souhaitée : si sa femme accouche d'un fils, il sera l'héritier universel ${ }^{32}$. De même dans le testament de 1514, ses trois fils sont ses héritiers universels à parts égales. Mais on observe une évolution entre les deux testaments en ce qui concerne la place réservée à son épouse ; dans le premier, étant donné que la venue d'un héritier mâle n'était pas encore assuré, elle a la possibilité de se remarier après le décès d'Alde, ou de rentrer au couvent ; ces possibilités lui sont retirées dans le deuxième testament, à un moment où le couple a déjà trois fils. Dans le premier cas, Alde prend des mesures pour que l'entreprise puisse perdurer, même en l'absence d'héritier direct, grâce au remariage de sa veuve ; dans le deuxième cas, il s'agit au contraire de préserver l'héritage des fils en essayant d'empêcher le remariage, et donc la perte de la dot substantielle de l'épouse. Le maintien de l'entreprise familiale détermine largement les stratégies élaborées par le père de famille, responsable de cette transmission.

L'espoir qu'Alde plaçait dans ses enfants, l'attente d'être père, n'est cependant pas uniquement à lire sous l'angle purement économique. Son attention au rôle de père est confirmé par les dispositions qu'il prend en faveur de sa fille, très jeune encore au moment de son testament de 1514, et pour laquelle il détaille les conditions de son éducation, avant son mariage ou son entrée au couvent ${ }^{33}$. Alde était un

29 M. MITTERRAUER, «Familie und Arbeitsorganisation in städtischen Gesellschaften des Mittelalters und der frühen Neuzeit », art. cit., p. 23-25.

30 F. ASCARELLI, M. MENATO, La Tipografia del '500 in Italia, op. cit., p. 337.

31 Le testament de 1506 est édité dans R. FULIN, «Documenti per servire alla storia della tipografia veneziana », $\mathrm{n}^{\circ}$ 155, Archivio Veneto, XXIX, 1882 ; le testament de 1514 est édité dans A. BASCHET, Aldo Manuzio. Lettres et documents 1495-1515, Venise, Antonellianis, 1867.

32 R. FULIN, « Documenti per servire alla storia della tipografia veneziana », art. cit.

33 «Preterea quia est mihi puella nomine Alda volo quod ipsa educetur inter monachas et sorores Sancti Francisi in eodem opido Carpi statuta mercede singulorum annorum sicuti videbitur Principibus predictis. Et cum nata fuerit annos sexdecim extrahatur exx monasterio praedicto et rogetur diligenter sine deceptione aliqua an velit esse religiosa et si persisterit in hoc bono proposito reducatur in idem monasterium et ei dentur ducatos 
humaniste, qui baignait dans les réflexions humanistes sur l'éducation et sur le rôle du père. Ces précisions ne sont donc pas étonnantes de sa part, mais témoignent bien d'une attention nouvelle portée à l'enfant et à son éducation ${ }^{34}$.

La transmission du patrimoine, et en particulier du capital matériel nécessaire à l'imprimerie, relève donc de stratégies de la part de l'imprimeur. Si celui-ci est père d'enfants issus d'une union légitime, la stratégie la plus évidente consiste à faire de son fils aîné le légataire universel ou à partager l'héritage entre les fils. L'absence d'enfants crée une situation fragile. Deux possibilités s'ouvrent alors: le remariage de la veuve selon des modalités précisées par le mari défunt ou la famille survivante ; la transmission en direction des proches qui savent eux-mêmes le métier d'imprimeur ou qui sont suffisamment insérés dans ce milieu pour pouvoir tirer profit de ce matériel. À cela s'ajoute, pour un père imprimeur, l'importance vitale de la formation, seule garante de la survie de l'entreprise dans le temps.

À travers la transmission du patrimoine de l'entreprise familiale, on voit donc également apparaître la question de l'apprentissage du métier et de la transmission du savoir-faire, des réseaux et du capital du père vers le fils. Ce qui m'amène désormais à m'intéresser à la place du père dans l'entreprise familiale, qui permet cette transmission du père au fils.

\section{Le père dans l'entreprise économique et entrepreneur de sa famille}

\section{L'organisation de l'atelier : l'exemple de l'atelier d'Alde Manuce}

Le père de famille, de son vivant, est le chef de l'entreprise familiale, son représentant légal, alors même que bien souvent, sa femme et certains de ses enfants travaillent sous sa direction. Reste à savoir de quelle manière cela se traduit concrètement dans l'organisation de l'atelier. Nous avons malheureusement assez peu de sources qui en témoignent. Le seul atelier pour lequel nous pouvons avoir un aperçu détaillé du fonctionnement concret est celui d'Alde Manuce. Les sources que nous avons à son sujet sont nombreuses et permettent d'être croisées assez aisément. Je m'appuie ici sur l'étude qui a été faite de l'imprimeur humaniste par Martin Lowry, en particulier à partir des témoignages d'Érasme qui a séjourné quelques temps chez Alde et son beau-père, Andrea Torresanus. Ce témoignage est écrit sous la forme d'un dialogue satirique extrêmement caustique, qui dresse un portrait fort peu élogieux de l'associé d'Alde, Andrea Torresani, tyran de l'ensemble de la maisonnée ${ }^{35}$.

Celle-ci est composée de la réunion de la famille d'Alde Manuce et d'Andrea Torresanus, à la suite du mariage d'Alde et de la fille d'Andrea, Maria. Érasme décrit une maisonnée d'une trentaine de personnes, en comptant les ouvriers, sans doute une quinzaine, les domestiques, la famille à proprement parler et huit ou neuf invités. Ce fonctionnement est habituel dans le milieu artisanal, où le maître a le plus souvent la charge du logement et de la nourriture de ses employés, tant ceux qui servent directement dans l'atelier, que les serviteurs qui s'occupent de la tenue de la maison. La hiérarchie de la famille, qui établit l'autorité du père de famille, se reporte dans le monde du travail, où le père a autorité sur les ouvriers et les domestiques. Érasme rapporte ainsi que le fonctionnement de l'atelier typographique mêlait tous les statuts dans le tumulte de l'activité, l'encre, les activités tant

tercenti pro ipsius vitae substentatione. Verum si amplius redere vouerit in monasterium, volo quod habeat pro dote ducatos sexcentos et vestes quas habuerit, et tunc nubat sicut videbitur Principibus praedictis et Andreae avo. » : A. BASCHET, Aldo Manuzio, op. cit..

$34 \quad$ Sur les évolutions de la paternité à la fin du Moyen Âge, voir notamment J. DELUMEAU, D. ROCHE, Histoire des pères et de la paternité, Paris, Larousse, 1990, en particulier chap. 2, «De Gerson à Montaigne, le pouvoir et l'amour »; ainsi que Cahiers de Recherches médiévales, «Être père à la fin du Moyen Âge », $\mathrm{n}^{\circ} 4$, 1997, numéro dirigé par D. LETT.

35 ÉRASME, «Opulentia sordida », Colloquia, Turin, Einaudi, 2002, p. 1202-1225. 
manuelles qu'intellectuelles; mais la hiérarchie était brusquement rétablie au moment du repas entre invités, famille et correcteurs d'un côté, et les ouvriers, qui mangeaient séparément, de l'autre ${ }^{36}$.

Dans le cas de la famille d'Alde Manuce, Érasme nous livre également le témoignage d'un exercice problématique de l'autorité du père de famille. En effet, qui est le père de famille et le chef de l'atelier: Alde Manuce ou Andrea Torresani, son beau-père ? Il semble avéré qu'Alde exerçait une autorité relativement tyrannique sur ses ouvriers et leur demandait une capacité de travail extrêmement importante, ce qui provoque des réactions et des protestations de leur part ${ }^{37}$. Cependant, la question de savoir qui dirigeait véritablement l'entreprise familiale, et partant, la famille, se pose. Érasme, dans son «Opulentia sordida », fait le portrait d'Alde comme un personnage entièrement sous la coupe de son beau-père : selon les termes de Martin Lowry qui résume bien la tonalité du dialogue, Érasme décrit Andrea Torresani comme un «arriviste grippe-sou, qui économise le combustible en déterrant de vieilles racines humides pour faire du feu, sert des coquillages trouvés dans les latrines publiques, des soupes à base de croûtes de fromages ou de tripes avariées, et du vin acide coupé d'eau. Ses hôtes souffrent de la pierre et ses fils tournent $\mathrm{mal}^{38}$. »

Il s'agit ici finalement d'une critique sous-jacente du mauvais père, par opposition au bon père de famille qui nourrit sa famille, veille à l'entretien de ses invités, et qui s'occupe de l'éducation et de la moralité de ses propres fils. L'auteur rapporte au contraire que les fils d'Andrea ne sont pas présents lors des dîners familiaux, préférant aller chercher meilleure chère ailleurs ; ce faisant, ils tournent mal et perdent aux dés des sommes que leur père refuse de dépenser pour ses hôtes ${ }^{39}$. À travers ce portrait, Érasme nous livre sa conception de la paternité : le père doit jouer un rôle à la fois d'économie domestique et d'éducation. Andrea Torresani échoue dans ces deux aspects.

Le problème réside en partie dans la présence de deux pères de familles qui doivent faire fonctionner l'entreprise familiale de concert. Celui qui a la prédominance ici, est celui qui a l'avantage de la fortune et de l'âge. Andrea Torresani est en effet celui qui a apporté les fonds au projet d'imprimerie humaniste et grecque d'Alde; il est celui qui tient les cordons de la bourse de la famille, de l'entreprise, et celui qui organise la gestion quotidienne de la maisonnée, sans qu'Alde n'ait véritablement son mot à dire à ce sujet.

Ce texte très riche nous renseigne sur l'exercice de l'autorité dans l'atelier d'imprimerie. Celle-ci peut être partagée entre la gestion concrète de l'activité productive et la gestion économique : Alde Manuce dirige les ouvriers dans l'atelier, Andrea Torresani en est le gestionnaire. Mais si cette bipartition peut exister, elle s'accompagne inévitablement d'une dépendance de l'un vis-à-vis de l'autre, une dépendance financière évidente. Si Alde est maître dans son atelier, son autorité tant dans l'entreprise que dans sa famille lui est déniée. La situation est sans doute assez similaire à celle existant entre les éditeurs et les imprimeurs à qui ils fournissent des fonds pour réaliser des éditions données. Cette situation était assez fréquente. Ainsi la plus grande partie de l'activité de Nicolas de Francfort, imprimeur et éditeur à Venise entre 1471 et 1516, consiste généralement à déléguer l'impression à d'autres imprimeurs. On a supposé qu'il a possédé pendant quelques années un atelier typographique, qui imprimait sous son nom propre, mais dont la direction technique était déléguée à un autre typographe, alors que Nicolas de Francfort assurait la gestion financière et commerciale ${ }^{40}$. Dans le cas d'une collaboration avec un autre imprimeur, la tutelle de l'éditeur qui fournit l'argent nécessaire sur l'imprimeur est tout aussi réelle.

\footnotetext{
36 M. LOWRY, Le Monde d'Alde Manuce. Imprimeurs, hommes d'affaires et intellectuels dans la Venise de la Renaissance, Évreux, Éditions du Cercle de la Librairie, 1989, p. 85-86.

$37 \quad$ Ibid., p. 104.

$38 \quad$ Ibid., p. 85-86.

39 ÉRASME, «Opulentia Sordida », op. cit., p. 1225 : «Utebantur, sed domi duntaxat, foris ligurienbant, scortabantur, ludebant aleam; quumque pater in gratiam honestissimorum convivarum graveretur teruntium impendere, iuvenes interdum una nocte sexaginta ducatos perdebant alea ».

40 Voir M. INFELISE, «Franckfordia (da Francoforte), Nicolo », dans Dizioniario Biografico degli Italiani, Rome, Treccani, 1998 et C. KIKUCHI, « Nicolas de Francfort, un Allemand à Venise : l'insertion d'un imprimeur dans la société vénitienne au tournant du XV siècle », Revue historique, à paraître.
} 


\section{Les apprentis}

Si l'on cherche à rentrer davantage dans le détail des rôles au sein de l'atelier, les sources sont plus rares. On comprend au détour d'un document les relations d'apprentissage qui pouvaient lier les uns et les autres. Cependant, il y a plus de suppositions que de certitudes sur les premiers imprimeurs.

À Venise pour le $\mathrm{XV}^{\mathrm{e}}$ siècle, l'un des seuls cas nous donnant quelques éléments est celui d'Evangelista, un imprimeur actif pendant un an seulement. Dans son colophon de 1482, on trouve cette mention: « éduqué dans cet art par l'illustre maître Nicolas Jenson, français, et par Gregorio Dalmate, et tous leurs associés ${ }^{41}$. » Dans le testament de Nicolas Jenson en 1480, on a effectivement la mention d'un Evangelista, qui était de la maisonnée du testateur, et à qui celui-ci lègue 25 ducats d'or en plus des gages qui lui étaient dus ${ }^{42}$. Dans le cadre d'un métier qui n'est pas formalisé, nous n'avons aucune indication des conditions d'apprentissage et de vie de ces apprentis ; mais l'on constate ici malgré tout qu'il s'agit bien de l'éducation prise en charge par les imprimeurs au sein de leur atelier et de la maisonnée, en échange d'un salaire. L'apprentissage d'Evangelista semble ici avoir été le fait de plusieurs imprimeurs à la suite, ainsi que de leurs associés respectifs ${ }^{43}$.

Nous pouvons également nous appuyer sur le cas d'Hanibal Foxius, qui fut apprenti de Christopher Valdarfer à Mila ; Valdarfer comme Foxius ont tous les deux exercé à Venise durant dans leur carrière. En 1476 est dressé un contrat d'apprentissage de deux ans pour «Annibale, fils de Filippo de Foxio du feu Andrea, de Parme », avec l'autorisation et la garantie du père. Annibale s'engage à travailler comme compositeur, à être fidèle et à habiter avec Valdarfer, contre un salaire de 50 lires $^{44}$. Le statut d'apprenti imprimeur n'étant pas encore bien réglé, à Venise comme à Milan, il est engagé sur le titre de compositeur alors qu'en réalité, il s'agit bien d'apprentissage ici.

À côté de ces apprentis extérieurs, des membres plus jeunes de la famille plus ou moins proche habitent fréquemment dans la même maisonnée. Il s'agit ici sans doute d'un apprentissage familial, comme on l'a déjà supposé en ce qui concerne les neveux des imprimeurs. Ainsi, le testament de Johann Manthem en 1474 mentionne «Gugliemus, mon cousin qui habite chez moi de présent ${ }^{45}$. » De même, dans le testament de Hermann Liechtenstein de 1494, on trouve mention de «Stuerinus, mon neveu qui se trouve de présent dans ma maison $»^{46}$.

Il faut souligner ici que dans ces deux cas, nous sommes dans le cadre d'une émigration de première génération. Les deux testateurs ont encore une grande partie de leur famille en Allemagne, comme en témoignent leurs testaments. Mais on peut supposer qu'ils ont éventuellement fait venir auprès d'eux un membre de leur famille élargie afin qu'il apprenne le métier d'imprimeur à leurs côtés, une fois qu'ils se sont eux-mêmes installés à Venise, à moins qu'ils n'aient émigré ensemble de leur ville d'origine.

À mesure que l'imprimerie se stabilise et se développe à Venise, on continue à retrouver des membres de la parentèle associée de près ou de loin aux imprimeurs. Mais de la même manière que pour la transmission de patrimoine, la place dans l'apprentissage de cette parentèle proche a été assez vite monopolisée par la présence de fils, parfois nombreux, qui fournissaient une main d'œuvre à éduquer et à qui transmettre le savoir-faire, en même temps que le capital.

Cela est particulièrement flagrant dans les cas où le fils est progressivement associé à la direction de l'entreprise familiale puisqu'il la reprend entièrement à la mort de son père, ce qui devient assez

41 L. BALSAMO, P. BELLTTINI (dir.), Anatomie bibliologiche: saggi di storia del libro per il centenario de «La Bibliofilía », Florence, Olschki, 1999, I, 94, je traduis.

42 ASV, Notarile, Testamenti, b. 68, $\mathrm{n}^{\circ} 263$ : «Item dimisti et legavit Evangeliste, qui est in domo cum ipso testatore, ducatos xxv auri ultra eius salarium quod habere debet, videlicet pro anno uno vel circa».

$43 \quad$ En effet, Gregorio Dalmate et Nicolas Jenson n'ont pas réalisé d'édition en commun.

44 T. RODEGLI MANNI, La Tipografia a Milano nel XV secolo, Florence, Olschki, p. 37.

$45 \quad$ ASV, Notarile, Testamenti, b. $481, \mathrm{n}^{\circ} 508$; je traduis.

46 ASV, Notarile, Testamenti, b. $66, \mathrm{n}^{\circ} 8$; je traduis. 
fréquent au cours du XVI ${ }^{\mathrm{e}}$ siècle. Andrea Torresanus par exemple associe progressivement son fils à l'imprimerie à partir de 1517, avant de lui laisser complètement l'entreprise familiale, avant même sa mort en $1529^{47}$. Un autre exemple d'association père-fils sur le long terme est celui de la famille Paganini que nous avons déjà évoquée. Paganino Paganini est originaire de Brescia. Il débute son activité à Venise en 1509. Entre 1511 et 1517, Alessandro, son fils, imprime à son propre compte à Venise. Puis entre 1519 et 1538, Paganino imprime à Toscolano, sur les rives du lac de Garde, peutêtre associé à son fils Alessandro. Père et fils sont à Venise en 1537-1538 où ils impriment sans doute ensemble le premier Coran arabe ${ }^{48}$. Nous n'avons pas de trace concrète de l'apprentissage de ces fils dans le cadre de l'atelier, mais il s'agit certainement d'une éducation complète, à la charge du père qui lui apprend le métier et toutes les compétences dont il aurait besoin par la suite : la lecture, l'écriture, les chiffres, la comptabilité, les langues éventuellement, notamment le latin. Il est difficile de savoir quelle part de cette éducation revenait effectivement au père. Mais l'association des pères avec leurs fils témoigne d'une volonté d'apprentissage rapide par la pratique concrète du métier.

Le père de famille chef d'un atelier familial a donc véritablement ici un rôle d'entrepreneur qui se doit de combiner stratégies familiales et économiques. La venue d'un aide de la famille proche permet de combiner à la fois les liens proches de la parenté, les liens de l'apprentissage et l'efficacité économique, qui était un enjeu majeur dans un secteur artisanal encore très fragile.

\section{Le père et ses enfants dans les stratégies familiales}

La permanence de l'entreprise implique que des mains capables puissent en hériter. Mais l'apprentissage technique ne fait pas tout. Dans une industrie où le partage des investissements et des risques a très tôt été vu comme une nécessité, le père a également un rôle actif dans le choix des alliances matrimoniales. Celles-ci sont essentielles pour l'entreprise familiale, puisqu'elles permettent d'augmenter le patrimoine de la famille, et partant le capital de l'entreprise. Elles permettent des alliances avec d'autres entreprises familiales du même type, ou avec des groupes sociaux pouvant avoir une utilité pour l'activité de la famille. Le père peut décider d'opter pour telle ou telle alliance pouvant être utile pour son entreprise; les enfants sous son autorité sont autant de possibilité pour agrandir l'entreprise ou faciliter l'activité.

Il est cependant difficile d'établir des liens directs. Des études du type de celle de Simona Cerutti sur les artisans de Turin aux $\mathrm{XVII}^{\mathrm{e}}$ et $\mathrm{XVIII}{ }^{\mathrm{e}}$ siècles qui permettent d'étudier très précisément les mariages, l'exogamie ou au contraire l'endogamie, sont infaisables ici compte tenu des sources ${ }^{49}$. De plus, postuler l'action directe d'un père ordonnateur serait sans doute réducteur et impliquerait d'une part une absence de latitude des enfants, d'autre part une rationalité tournée vers le seul but économique. Malgré ces précautions, nous pouvons malgré tout voir apparaître des modèles et des stratégies récurrentes.

Le premier type de stratégie consiste d'abord à privilégier le partage des risques économiques au sein du monde du livre, et donc à s'allier par le mariage avec une autre famille d'imprimeur. Cette stratégie est notamment celle adoptée par Alde Manuce qui se marie avec la fille de son associé, Andrea Torresani. C'est également celle de Francesco Renner, imprimeur allemand dont la fille Christina épouse le libraire Francesco de Madiis ${ }^{50}$; et enfin celle de Paganino de Paganini dont le fils Alessandro épouse Daria Rusconi, fille de Giorgio Rusconi, imprimeur milanais exerçant à Venise ${ }^{51}$.

47 F. ASCARELLI, M. MENATO, La Tipografia del '500 in Italia, op. cit., p. 333.

48 A. NUOVO, Alessandro Paganino, op. cit. et F. ASCARELLI, M. MENATO, La Tipografia del '500 in Italia, op. cit., p. 341-342.

49 S. CERUTTI, La Ville et les métiers : naissance d'un langage corporatif, Paris, France, Éditions de l'École des hautes études en sciences sociales, 1990.

$50 \quad$ R. RIDOLFI, «Francesco della Fontana, stampatore e libraio a Venezia », art. cit. Voir également le testament de Cristina Fontana : ASV, Notarile, Testamenti, b. 1153.

$51 \quad$ A. NUOVO, Alessandro Paganino, op. cit, p. 249. 
Ce sont donc ici des mariages très fortement endogames, non seulement au sein du même milieu socio-économique mais au sein de la même industrie.

Par ailleurs, on observe également des stratégies exogames qui cherchent à sortir du milieu du livre, cherchant parfois d'autres voies d'ascension sociale et d'insertion de la famille dans la ville. Ainsi Nicolas de Francfort se remarie avec Andriana da cha da Mestre, fille de riches verriers de Murano, et marie sa fille Paula à un riche marchand d'épices, Jacomo Bassani da Fontana; le résultat se voit notamment dans les investissements fonciers et immobiliers de Nicolas de Francfort ${ }^{52}$, qui ont sans doute été largement favorisés par sa belle-famille, mais également dans la dot que Jacomo Bassani da Fontana accorde à sa fille, la petite-fille de Nicolas de Francfort : une dot de 800 ducats qui représente une grosse somme dans un milieu d'artisans et de boutiquiers et qui les rapproche de l'élite marchande vénitienne $e^{53}$.

Les stratégies adoptées par les pères de famille ne sont donc pas univoques mais on y retrouve des logiques bien reconnaissables. Leurs propres mariages, comme ceux de leurs enfants, sont utilisés comme autant de moyens de faire prospérer l'entreprise familiale et la famille elle-même.

\section{Une répartition des rôles entre père et mère ?}

Pour conclure cette réflexion sur le rôle des pères dans les entreprises typographiques vénitiennes, il nous a semblé important de tenter une rapide comparaison avec le rôle des mères dans ces mêmes entreprises.

Il convient tout d'abord de souligner qu'il n'existe que de très rares exceptions à Venise où une femme dirige l'entreprise. Entre 1468 et 1530, on n'en compte que deux. La première, Anna Notaras, éditrice en 1499, est la fille de Lucas Notaras, amiral et méga-duc de Constantinople. Réfugiée en Italie, elle subventionne le développement des presses grecques à Venise ${ }^{54}$. Mais il s'agit ici véritable d'un mécénat et sa situation, du fait de son origine illustre, est véritablement exceptionnelle. D'autre part, Elisabeth Rusconi est la veuve de Giorgio Rusconi, imprimeur milanais qui exerce à Venise entre 1500 et 1521 . On trouve d'abord des éditions aux noms des deux fils de Giorgio, puis Elisabeth signe ellemême, peut-être suite à un désaccord avec les deux héritiers ${ }^{55}$.

En ce qui concerne le rôle des femmes dans l'atelier et le commerce du livre, celui-ci a été en particulier bien étudié pour l'époque moderne dans des villes comme Paris ou Lyon ${ }^{56}$. Pour l'Italie, Deborah Parker souligne le fait que les femmes typographes peuvent à l'occasion reprendre l'entreprise de leur mari défunt, mais qu'il s'agit là bien d'une situation exceptionnelle voire anormale $^{57}$. Cependant, les épouses d'imprimeur ont une importance centrale dans le fonctionnement même de l'atelier et dans l'éducation de leurs enfants, en particulier quand ceux-ci sont amenés à reprendre l'entreprise familiale. La figure de Maria Torresani a en particulier été mise en valeur dans

$52 \quad$ Cela apparaît notamment dans ses deux testaments de 1519 et 1524 ainsi que dans sa déclaration pour les Decime de 1514 : ASV, Notarile, Testamenti, b. 66, n 320 ; ASV, Cancellaria inferiore, Miscellanea, b. 29, $\mathrm{n}^{\circ} 2936$; ASV, Savi Decime - 1514, b. 24, n 4 .

$53 \quad$ Voir le testament de Jacomo Fontana de 1528 : ASV, Cancellaria inferiore, Miscellanea, b. $29, \mathrm{n}^{\circ} 2950$. Pour plus de précisions, voir C. KIKUCHI, « Nicolas de Francfort, un Allemand à Venise : l'insertion d'un imprimeur dans la société vénitienne au tournant $\mathrm{du} \mathrm{XV}$ siècle », art. cit. à paraître.

54 D. M. NICOL, «Anna Notaras Palaiologina», The Byzantine Lady. Ten Portraits, 1250-1500, Cambridge, Cambridge University Press, 1994.

55 F. NOVATI, « Donne tipografe nel Cinquecento », Il Libro e la stampa, 7, 1907, p. 42-32.

$56 \quad$ Voir en particulier S. POSTEL-LECOQ, «Femmes et presses à Paris au XVI siècle : quelques exemples », Le Livre dans l'Europe de la Renaissance: actes du XXVIII colloque international d'études humanistes de Tours, P. AQUILON, H-J MARTIN (dir.), Tours, Promodis, 1988 ; N. ZEMON DAVIS, «Women in the Crafts in Sixteenth-Century Lyon », Feminist Studies, n 8, p. 46-80 ; S. LENKEY, "Printers' Wives in the Age of Humanism », Gutemberg-Jahrbuch, 1975, p. 331-337

57 D. PARKER, «Women in the Book Trade in Italy, 1475-1620 », Renaissance Quaterly, 49.3, 1996, p. 509-541, p. 534. 
la littérature : pourtant elle n'est que très rarement mentionnée dans les sources, sa présence est d'une extrême discrétion. Mais en tant que fille, épouse et mère d'imprimeurs, on a supposé qu'elle avait dû acquérir non seulement une éducation solide, mais également une connaissance pratique du fonctionnement d'un atelier typographique et de la réalisation d'une édition. Son fils, Paulo Manuzio, devient également un imprimeur humaniste et un lettré célébré, ce qui suppose une éducation attentive de la part de la mère ; Paulo n'avait en effet que deux ans à la mort de son père ${ }^{58}$.

Le cas de Maria Torresanus est malgré tout très délicat, en l'absence de sources qui nous permettraient d'évaluer son rôle effective. D'autres cas sont plus facilement observables, en particulier des cas où l'on observe une mère mener délibérément une stratégie en vue du placement de ses enfants et de la continuité de l'entreprise. Les études sur l'imprimerie vénitienne se sont en particulier intéressées à Paula de Messine, veuve de Johann de Spire, premier imprimeur de la ville. Celle-ci se remarie avec Johann de Cologne, ce qui avait rendu possible la continuité du capital entre les deux hommes. Cette même stratégie se retrouve dans le mariage de sa fille, Hieronyma, fille de Jean de Spire. Celle-ci épouse un associé de Johann de Cologne, Gaspar de Dinslach. Enfin, on peut voir une stratégie d'ancrage dans la société vénitienne avec le mariage d'une des filles de Hieronyma avec Giovanni Barbarigo, membre d'une grande famille patricienne de Venise ${ }^{59}$. Il s'agit ici pour ces deux femmes de permettre la continuité de l'entreprise familiale, mais aussi une ascension sociale fulgurante, qui allie la famille du premier imprimeur de Venise au patriciat de la ville.

Cependant, ces cas où l'on voit la mère s'octroyer le rôle dévolu au père dans l'organisation de la famille sont souvent conflictuels; la famille et les proches l'acceptent souvent difficilement. Ainsi, dans le cas de Paula de Messine, on observe à travers ses testaments que les relations avec ses enfants se détériorent au cours du temps ; chaque enfant est déshérité, l'un après l'autre ; pour finir, lors de son dernier testament en 1488, elle demande clairement à ce que ses enfants, fils comme fille, ne reçoivent rien d'elle rien car, dit-elle, ils la persécutent ${ }^{60}$. Un autre exemple est éloquent : au moment de recevoir la tutelle de ses frères et sœurs encore mineurs, Cristina Fontana, fille, veuve et sœur d'imprimeurs ou de libraires, se voit attaquée en justice par un membre de sa famille paternelle, Bernardino Fontana; si elle obtient finalement gain de cause, on voit ici que le rôle de chef de famille qui lui était attribué ne faisait pas l'unanimité parmi les hommes de son entourage ${ }^{61}$.

Le père reste malgré tout celui qui dirige le travail de l'atelier et place ses enfants, éventuellement selon une stratégie déterminée pour le bien social et économique familial ; l'autorité de la mère est vécu comme une transgression de la norme, plus ou moins bien supportée. S'il existe une répartition des rôles entre le père et la mère, que nous percevons mal dans l'atelier ou dans l'éducation des enfants, elle reste difficile à comprendre et à analyser, si ce n'est par les conflits qu'ils provoquent quand la distinction genrée n'est pas respectée.

J'ai tenté ici d'étudier les débuts de l'imprimerie à Venise sous l'angle de la paternité, afin de faire mieux ressortir certains de ces fonctionnements, et afin de voir les évolutions que connaît la paternité dans les milieux urbains à la fin du Moyen Âge. Cette situation permet de comprendre l'importance croissante que prend la paternité pour les acteurs du point de vue de l'activité économique. Avec la stabilisation des imprimeurs migrants en ville, la création de conditions économiques stables, on voit apparaître des enfants dans les sources, qui sont l'objet d'une attention croissante de la part des pères.

\footnotetext{
$58 \quad$ Ibid., p. 531-532.

$59 \quad$ Voir à ce sujet G. LUDWIG, «Antonello da Messina und Deutsche und Niederländische Künstler in Venedig », Jahrbuch der Königlich Preussische Kunstsammlungen, 1902, p. 43-65 et P. BRAUNSTEIN, «Les Allemands et la naissance de l'imprimerie », art. cit.

60 ASV, Notarile, Testamenti, b. 718, $\mathrm{n}^{\circ}$ 179, édité dans G. LUDWIG, «Antonello da Messina und Deutsche und Niederländische Künsteler in Venedig », art. cit. : «Filiis autem meis quia me persecuti sunt, nec filiee mee nihil reliquo de meis bonis, quibus tamen Deus ignoscat; quibus et etiam ego ignosco ».

61 ASV, Giudici del Proprio, Sentenze a legge, b. 12, fol. 24 et suivants, 1490. Pour plus de précisions sur le rôle des femmes dans l'industrie du livre vénitien, on pourra consulter C. KIKUCHI, «Les Femmes dans le milieu du livre vénitien, fin $\mathrm{XV}^{\mathrm{e}}$-début XVI $\mathrm{X}^{\mathrm{e}}$ siècles », journée d'études «Acteurs sociaux en situation « Europe. Fin du Moyen Âge) », 22 mars 2014, Paris-Sorbonne - UMR 8596.
} 
La transmission du père au fils prend une importance de plus en plus grande : transmission de capital, mais aussi transmission de savoir-faire, dans le cadre de l'atelier dont le père est le chef. L'autorité du père s'exerce sur le fils en tant que père, mais aussi en tant que maître sur son apprenti.

Les évolutions concernant l'exercice de la paternité dans le milieu du livre vénitien au $\mathrm{XV}^{\mathrm{e}}$ siècle et au début $\mathrm{du} \mathrm{XVI}^{\mathrm{e}}$ siècle, et que l'on peut particulièrement observer à travers les testaments de ces imprimeurs, permettent également de mieux comprendre l'implantation de ce nouvel art dans la ville et les modalités de son insertion. Il permet en particulier de comprendre que la transmission patrilinéaire de l'entreprise typographique ne va pas de soi, mais est au contraire le fruit d'évolutions sociales et de stratégies de la part de la famille, plus particulièrement du père. Lorsque ces stratégies fonctionnent, elles permettent la constitution des grandes dynasties qui ont retenu l'attention des historiens et des bibliophiles. 